\title{
Phase-Coupled Oscillations in the Brain: Nonlinear Phenomena in Cellular Signalling
}

\author{
Vikas Rai, ${ }^{1}$ Sreenivasan Rajamoni Nadar, ${ }^{2}$ and Riaz A. Khan ${ }^{3}$ \\ ${ }^{1}$ School of Computer and Systems Sciences, Jawaharlal Nehru University, New Delhi 110067, India \\ ${ }^{2}$ Division of Neuroradiology, The Russell H. Morgan Department of Radiology and Radiological Science, School of Medicine, \\ The Johns Hopkins University, Baltimore, MD 21287, USA \\ ${ }^{3}$ Department of Medicinal Chemistry, College of Pharmacy, Qassim University, Qassim 51452, Saudi Arabia
}

Correspondence should be addressed to Sreenivasan Rajamoni Nadar; sreenivasan.r.nadar@gmail.com

Received 16 January 2013; Accepted 20 February 2013

Academic Editors: J. Chow, J. Crezee, and J. H. Wu

Copyright (C) 2013 Vikas Rai et al. This is an open access article distributed under the Creative Commons Attribution License, which permits unrestricted use, distribution, and reproduction in any medium, provided the original work is properly cited.

\begin{abstract}
We report the existence of phase-coupled oscillations in a model neural system. The model consists of a group of excitatory principal cells in interaction with local inhibitory interneurons. The voltages across the membranes of excitatory cells are governed primarily by calcium and potassium ion conductivities. The number of potassium channels open at any given instant changes in accordance with a deterministic law. The time scale of this change is set by a constant which depends on midpoint potentials at which potassium and calcium currents are half-activated. The growth of mean membrane potential of excitatory principal cells is controlled by that of the inhibitory interneurons. Nonlinear oscillatory system associated with these limit cycles starting from two different initial conditions maintain a definite phase relationship. The phase-coupled oscillations in electrical activity of the neuronal cells carry together amplitude, phase, and time information for cellular signaling. This mechanism supports an energy efficient way of information processing in the central nervous system. The information content is encoded as persistent periodic oscillations represented by stable limit cycles in the phase space.
\end{abstract}

\section{Introduction}

The rhythmic oscillations are abundant in nature and they also exist at all levels of organization in the biotic world. The well-known examples are circadian rhythms [1], relaxation oscillations in heart beats $[2,3]$, and gene regulatory networks [4]. The electrical and magnetic activity of human brain has many oscillatory networks that coexist, and they perform unique physiological and cognitive functions. The frequency of oscillations in these networks is used to categorize them in different bands such as $\alpha, \beta, \gamma$, and $\delta$ [5], and they characterize different physiological states. The oscillations in $\alpha$ band represent eyes closed/relaxed states, the $\beta$ band is characteristic of eyes open, cognition is and alert state, $\gamma$ during higher cognitive functioning, and $\delta$ is during sleep condition or under the action of anesthesia. These oscillations represent ongoing and spontaneous activities of the brain as under process by different neuronal organizations emerging from arrays of neuronal tissues and cells in the brain. The neuronal cells communicate with each other through action potentials as stated in electrophysiological parlance. These action potentials are rapid reversals in voltages across the plasma membrane of neuronal cells axons, mediated by voltage-gated ion channels [6]. Brain oscillatory networks play a key role in information processing, and they are characterized by their frequency, amplitude, and phase.

Nerve cells, or neurons, are responsible for receiving and transmitting signals to and from the central nervous system. Information is encoded and transmitted as spikes in membrane electrical potential called action potentials. Transmembrane electrical potential is due to ion currents and the capacitance of the cell membrane. The four-dimensional Hodgkin-Huxley system [7] represents the process by which action potentials are generated in the giant axon of the squid [8]. All excitation phenomena this model exhibits can be visualized in two dimensions. Morris-Lecar (ML) representation [9] shows such visualization as having two noninactivating voltage sensitive conductances. It is assumed that $\mathrm{Na}^{+}$ 
conductivity is exceptionally high in comparison to $\mathrm{Ca}^{++}$and $\mathrm{K}^{+}$conductances in the neuronal cells of pyramidal type. Apart from excitatory pyramidal neuronal cells, the brain contains diverse classes of specialized neuronal cells which are characterized as inhibitory interneurons selectively connecting to specific parts of the somatic-dendritic surfaces of pyramidal neuronal cells and other interneurons in the brain. These inhibitory interneurons provide necessary autonomy and independence to neighboring pyramidal neuronal cells. The opposing actions of excitation and inhibition of different types of neuronal cells give rise to membrane and network oscillations which, in turn, provide temporal coordination among the messages conveyed by excitatory/principal cells. Thus, the inhibitory interneurons also play a specialized role in generating oscillatory patterns at different frequencies.

In this paper, we report that the system supports a new kind of oscillation in a population of excitatory neuronal cells interacting with local interneurons. These oscillations are phase preserving and originate via Neimark-Sacker bifurcations.

\section{The Model}

The interaction between population of excitatory principal cells and inhibitory interneurons has been modeled by the following system of nonlinear differential equations [10]:

$$
\begin{gathered}
\frac{d V}{d t}=-g_{\mathrm{Ca}} m_{\infty}(V-1)-g_{K} W\left(V-V^{K}\right) \\
-g_{L}\left(V-V^{L}\right)+I-\alpha_{\mathrm{inh}}(Z) Z, \\
\frac{d W}{d t}=\frac{\phi\left(W_{\infty}-W\right)}{\tau_{W}}, \\
\frac{d Z}{d t}=b\left(c I+\alpha_{\mathrm{exc}}(V) V\right),
\end{gathered}
$$

where $V$ is the mean membrane potential of the principal cells, $W$ is the fraction of open potassium channels at any point of time, and $Z$, is the mean membrane potential of the local inhibitory interneurons. $\alpha_{\text {inh }}$ is the synaptic strength of inhibitory interneurons. $\alpha_{\text {exc }}$ is that of the excitatory principal cells. $I$ is the constant external current fed into to the system. The presence of parameter $b$ in the last equation signifies that the time scale of inhibitory cells is different from that of $V, W$. The parameter $c$ measures the strength of the feed-forward inhibition. The detailed meanings of other parameters are given in our earlier work [10].

The last equation was added to the ML system to describe the effect of population of inhibitory interneurons synapsing on the pyramidal cells. The model describes temporal dynamics of population of excitatory principal cells which are in interaction with inhibitory interneurons. Both kinds of cells are fed with external current. This helps us to investigate the effects of disinhibition on the model dynamics. The communities of on-off excitatory and inhibitory synapses were modeled using tangent hyperbolic functions. These functions provide a novel way to represent collective behavior of a large number of channels with open/closed and on/off characteristics. These functions are given by the following equations:

$$
\begin{aligned}
& \alpha_{\text {exc }}(V)=\alpha_{\text {exc }}\left[1+\tan h\left(\frac{V-V_{5}}{V_{6}}\right)\right], \\
& \alpha_{\text {inh }}(Z)=\alpha_{\text {inh }}\left[1+\tan h\left(\frac{Z-V_{7}}{V_{6}}\right)\right],
\end{aligned}
$$

where $V_{5}$ and $V_{7}$ are thresholds for the synaptic activity functions of the excitatory principal cells and inhibitory interneurons. $V_{6}$ is the steepness parameter for both the functions.

\section{Origin of Phase-Coupled Oscillations: A Geometric Theory}

3.1. Phase Plane with Fast and Slow Time Scales. The dynamics of a system of equations are determined by its vector field. The phase plane methods focus on the direction of vector fields in the vicinity of critical points. To construct the phase plane of systems with two different time scales, geometric theory of singular perturbation is employed. Equations (1) and (2) constitute the single neuron ML system. The action of a group of local interneurons is modeled by equation (3), wherein parameter $b$ is a temperature scaling factor and ensures that the action of interneurons is on a slower time scale than that of the subsystem constituted by movements of calcium and potassium ions. The geometric theory of singular perturbations $[11,12]$ is a powerful tool for analyzing highdimensional systems with two different time scales. It offers a reduction of the dimensionality of the original system in order to study the essential dynamics.

3.2. Local Bifurcations in the Vector Field. Bifurcations determine the properties of the cell. We mention a few codimension 1 bifurcations which occur in higher dimensional systems. When the rest state is near a saddle-node bifurcation, the cell fires spikes with arbitrary low frequency. The cell acts as an integrator. On the other hand, in the situation when the rest state is near an Andronov-Hopf bifurcation, the cell fires in a certain frequency range; its spikes are called all-or-none events $[13,14]$. It can fire in response to an inhibitory pulse and it acts as a resonator; it responds preferentially to a certain (resonant) frequency of the input. Bifurcations which result in sharp loss of stability are saddle-focus homoclinic orbit, subcritical flip, subcritical Neimark-Sacker, fold limit cycle on homoclinic torus, and the blue-sky bifurcations. Subcritical Neimark-Sacker bifurcations are implicated for the birth of phase-coupled oscillations. In this type of bifurcation, an unstable invariant torus shrinks down to a stable limit cycle.

\section{Simulation}

A previous study [10] suggested that two synaptic strengths are the key parameters in deciding the mode of signalling and the information transport used by cortical neurons in different experimental situations with the value of the stimulus current fixed at $=0.3$. Base parameter set for which 


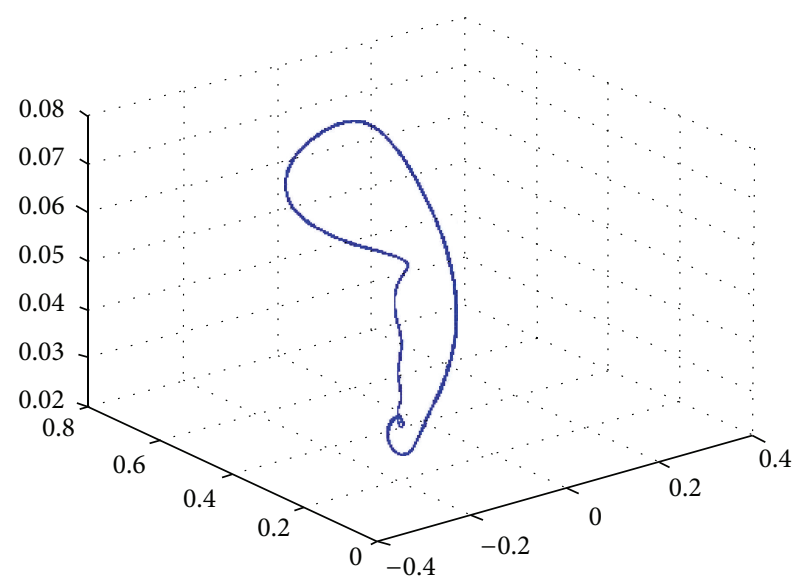

(a)

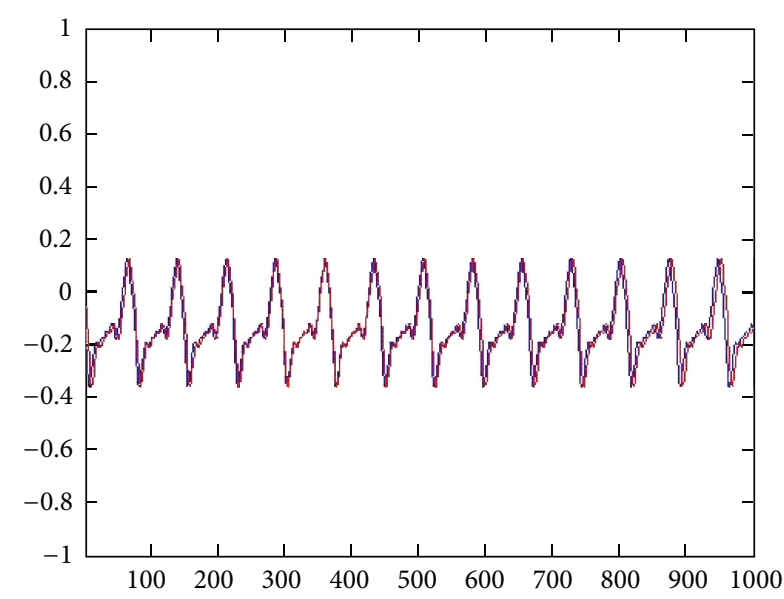

(b)

FIGURE 1: Sustained spiking activity observed at $\alpha_{\text {inh }}=1.2, \alpha_{\text {exc }}=0.8$. Signals originating from two nearby points overlap with each other. (a) is the phase space and the (b) is the time series of two nearby initial conditions. The phase information is lost by the action of dissipative forces in the system as mean membrane potential time series for two nearby initial conditions overlap.

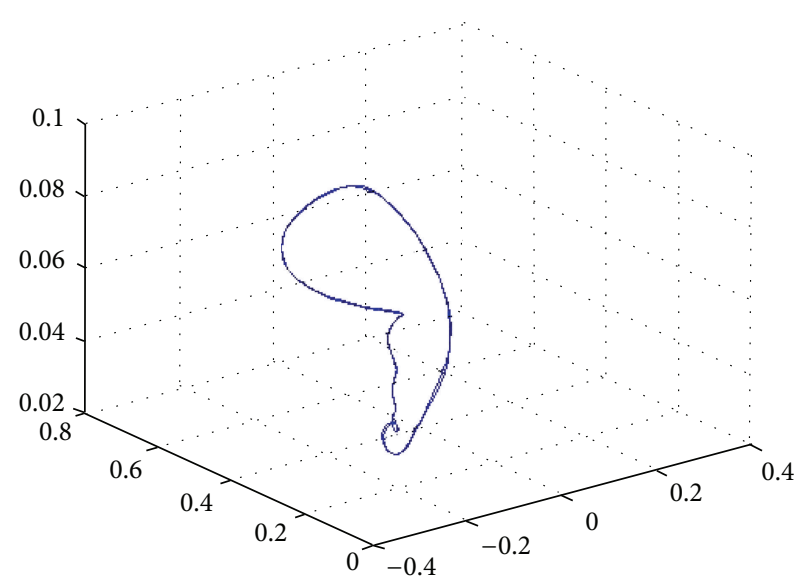

(a)

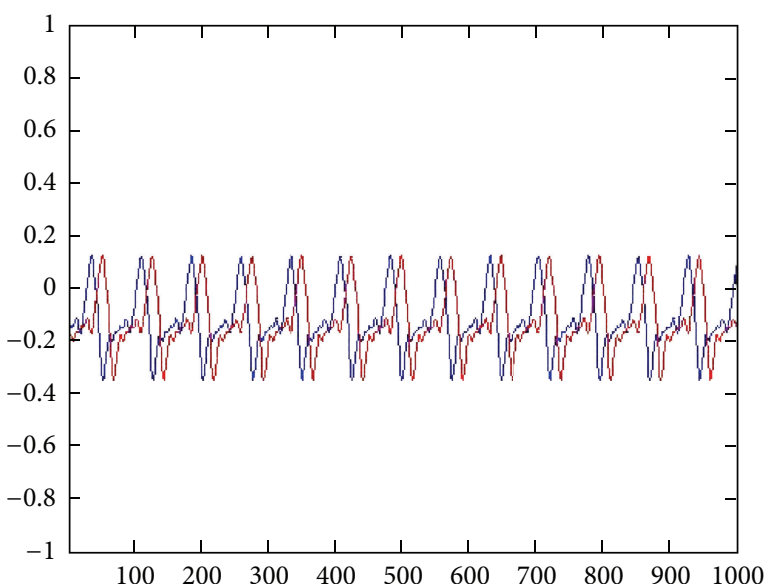

(b)

FIGURE 2: Sustained spiking activity observed at $\alpha_{\text {inh }}=1.1, \alpha_{\text {exc }}=0.8$; signals originating from two nearby initial conditions do not overlap. (a) is the phase space and (b) is the time series of two nearby initial conditions (close membrane potentials). The phase information is retained as two signals maintain their identity.

the model system (given by (1), (2), and (3)) displayed sustained spiking activity were as follows:

$V_{1}=-0.01, V_{2}=0.15, V_{3}=0.03, V_{4}=0.3, V_{5}=0.0, V_{6}=$ $0.04, V_{7}=0.05, V^{K}=-0.7, V^{L}=-0.5, \phi=0.4, I=0.3 ; b=0.15$, $c=0.18, g_{\mathrm{Ca}}=1.1, g_{L}=1.0, \alpha_{\mathrm{exc}}=1.0$, and $\alpha_{\mathrm{inh}}=1.0$.

The two-dimensional parameter scans were performed in $\alpha_{\text {exc }}$ and $\alpha_{\text {inh }}$ parameter spaces in the range 0.7 to 1.3 with step size 0.1. $\alpha_{\mathrm{exc}}$ and $\alpha_{\mathrm{inh}}$ are constants which parameterized the synaptic strengths of inhibitory interneurons and excitatory pyramidal cells, respectively. These ranges were chosen on the basis of system dynamics [10] which provided an insight into the network dynamics at a particular node in the hippocampus and the way it was affected by changes in synaptic strengths.

The systems of coupled nonlinear ordinary differential equations were simulated on MATLAB at base value of the parameters. The simulation experiments were performed by varying the synaptic strengths of excitatory pyramidal cells on the inhibitory interneurons and vice versa. Figures 14 summarize different kinds of signals of electrical activity observed in the excitatory-inhibitory system. The persistent periodic oscillations which represent the human brain in states of resting or activity are presented in Figure 1. This displays spiking of action potentials showcasing brain activity under the action of dissipative forces. We report a new kind of self-sustained oscillations which preserve phase information. These oscillations were observed during parameter scans aimed at thorough investigation of dynamics by varying the strength of either of the synapses. These new kinds of oscillations represent induced activity which is in response to a stimulus, for example, event-related evoked potentials obtained from the electroencephalogram by stimulus-locked 


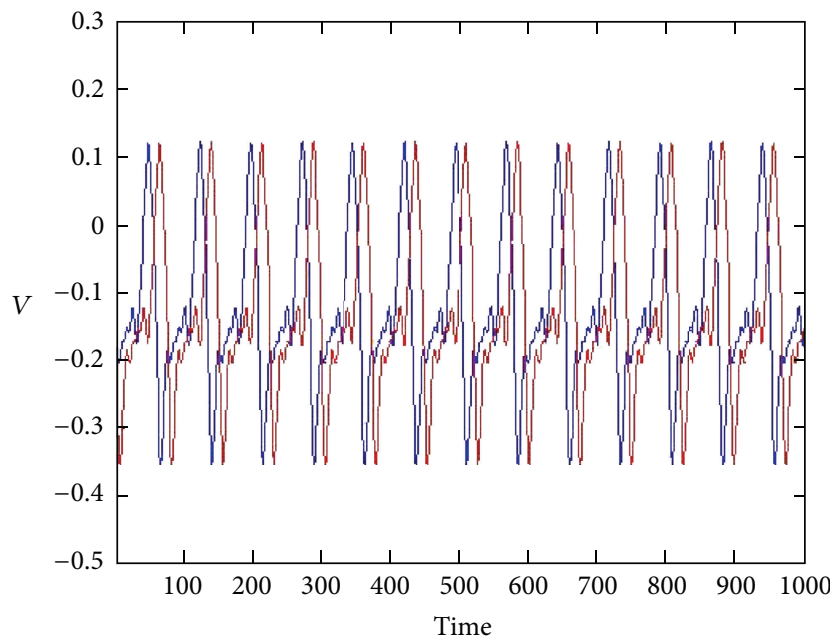

(a)

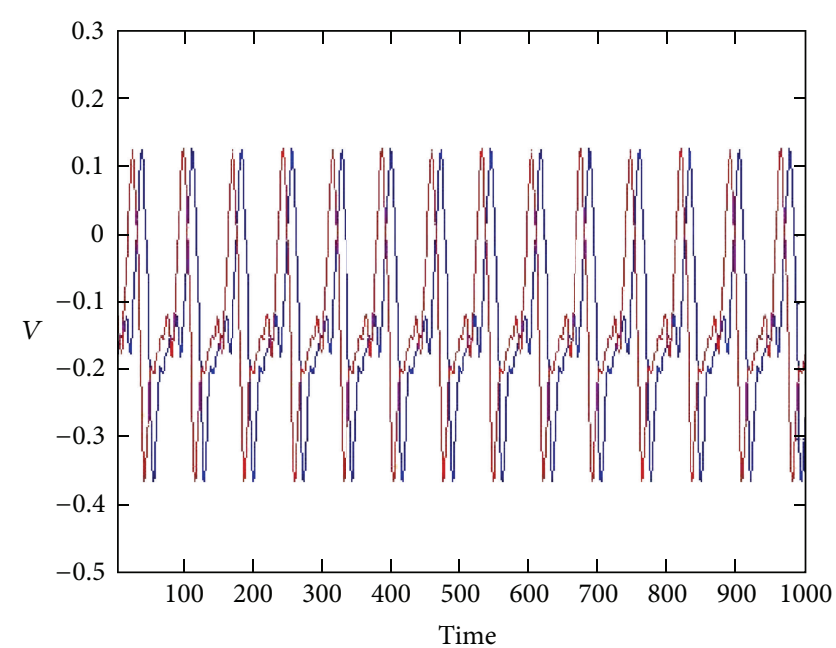

(b)

FIGURE 3: Time series for two initial conditions which differ in mean membrane potential by 0.01 (relative units) computed for parameter values $\alpha_{\text {inh }}, \alpha_{\text {exc }}=0.8$. (b) shows the time series for the mean membrane potentials for $\alpha_{\text {inh }}=1.3, \alpha_{\text {exc }}=0.8$ where blue and red coloured signals represent variations in the mean membrane potential for the two nearby initial conditions. In (a), the blue signal precedes the red one and the reverse is the case in (b).

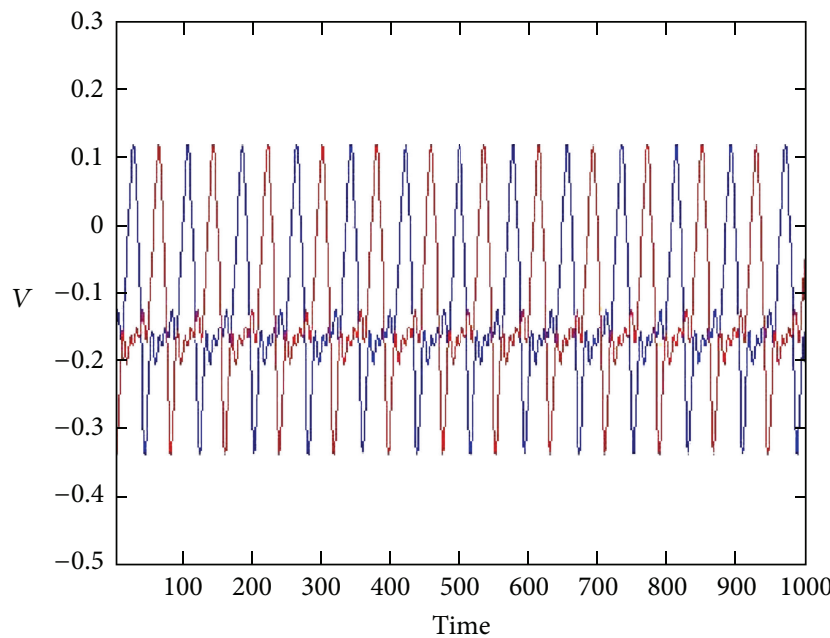

(a)

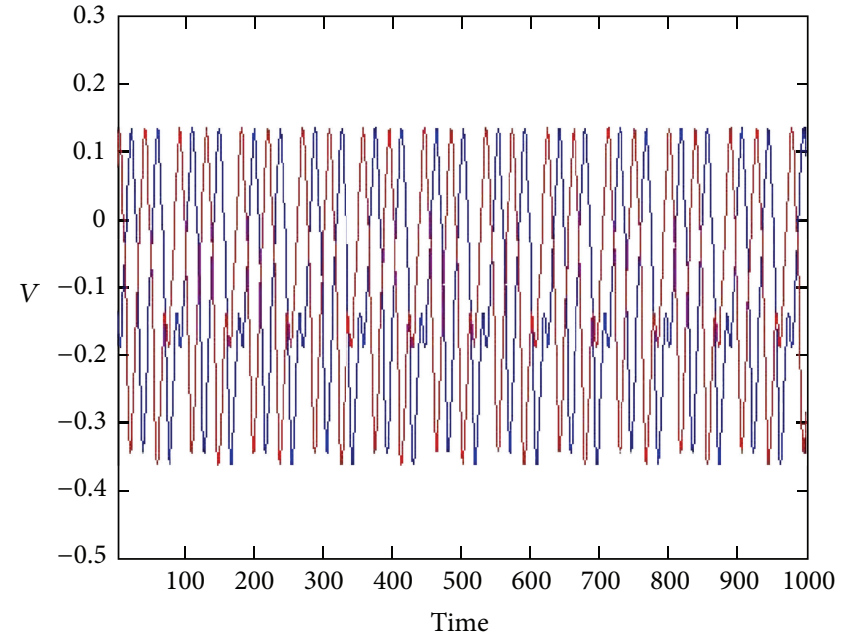

(b)

FIGURE 4: (a) shows the time series of the mean membrane potential of the pyramidal cell for two close initial conditions computed at $\alpha_{\text {inh }}=$ $0.7, \alpha_{\text {exc }}=0.8$, where the signals have the same amplitude but the phase is different. (b) shows the time series of mean membrane potentials at $\alpha_{\mathrm{inh}}=0.7, \alpha_{\mathrm{exc}}=1.1$.

averaging. The phase information is preserved when two time series for mean membrane potential starting from nearby initial conditions do not overlap. The phase-coupled limit cycles, which carry information about the initial conditions (mean membrane potentials of two neuronal columns which are nearby in space), were detected in the system at different sets of values of synaptic strengths. The two stable oscillations maintain a definite phase relationship. The coupled oscillatory system starting from two different initial conditions, maintaining a definite phase relationship wherein one signal phase is ahead or behind the other asymptotically. In this paper, we denote the phase ahead of time as PCO $(+)$ and reverse case as PCO $(-)$. The two oscillations are represented in Figure 3.

The neuronal cells in hippocampus fire persistently in response to brief stimulus and typical time span for this activity is few seconds. The observations [15] involving "delay neurons" in nonhuman primates suggest that the persistent activity associated with WM hippocampus is important for WM when multiple items are being processed. These results can be best understood in terms of output of the present mathematical model. During processing of electrical activity 


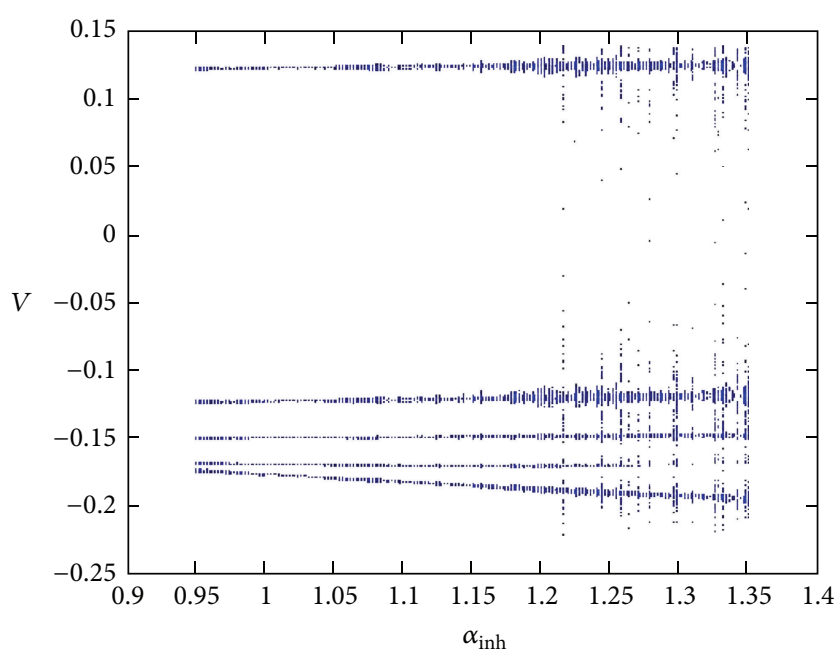

FIGURE 5: Bifurcation diagram treating $\alpha_{\text {inh }}$ as bifurcation parameter. $\alpha_{\text {exc }}=0.8$ and other parameters were fixed at their base value.

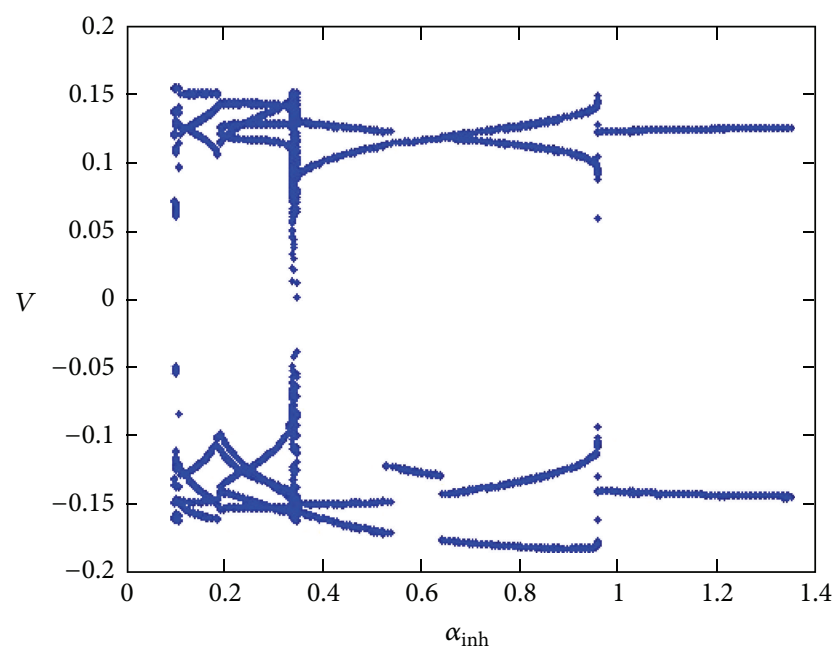

FIGURE 6: Bifurcation diagram treating $\alpha_{\text {inh }}$ as bifurcation parameter. $\alpha_{\text {exc }}=0.95$ and other parameters were fixed at their base value.

of neurons, a new set of oscillations was discovered. These oscillations which were represented by stable limit cycles in the phase space of the system signify persistent activity. The analysis established that oscillations in the mean membrane potential are phase coupled.

The hippocampus employs these quadruplet signals (cf. Figure 4) when the brain handles more than one task [16]. In computational analyses, PCOs (doublets and quadruplets) are represented by stable limit cycles of longer periods (complex periodic orbits) in the phase space of the relevant dynamical system. The phase coupled blue and red signals bifurcate and give birth to another phase-coupled oscillations.

In order to establish that these oscillations are different from oscillations on a regular stable limit cycles, we compute bifurcation diagrams treating $\alpha_{\text {inh }}$ as the bifurcation/control parameter. In Figure 5, the period-doubling bifurcations are

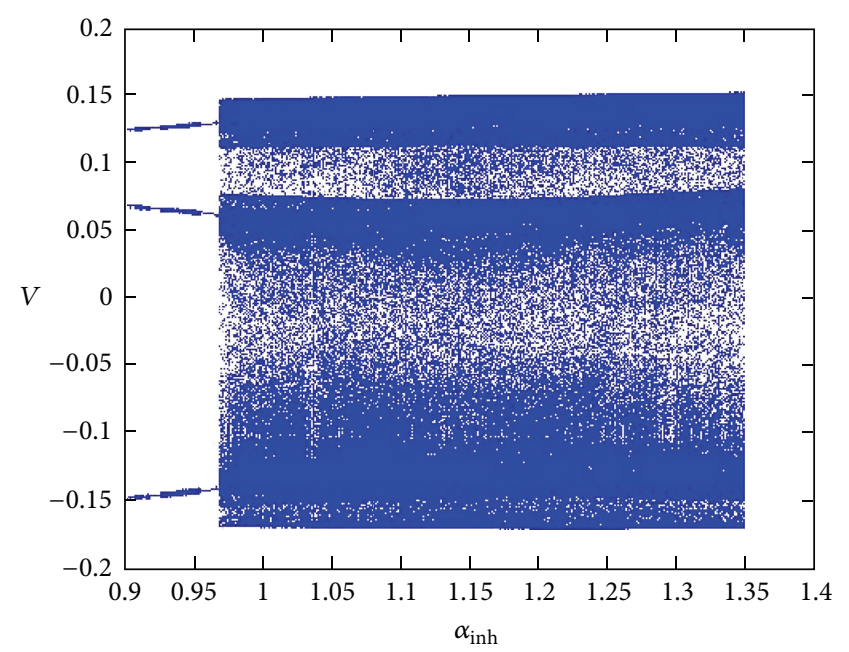

FIGURE 7: Bifurcation diagram treating $\alpha_{\text {inh }}$ as bifurcation parameter. $\alpha_{\text {exc }}=1.0$ and other parameters were fixed at their base value.

not visible, but when the value of the synaptic strength of the excitatory neuronal cells is increased, period doubling bifurcations appear but are repeatedly interrupted (cf. Figure 6). However, when this parameter is further increased, period doubling and halving sequences disappear. Figure 7 suggests that stable limit cycles are phase preserving and they exist in appreciable ranges of two synaptic strengths.

\section{Discussion}

A stable limit cycle in phase space suggested existence of a dissipation mechanism when the system is subjected to large amplitude perturbations (changes in initial conditions in case of a model system such as those studied in the present paper). The pumping of energy into the system starts when amplitude of oscillations becomes small. The presence of a stable limit cycle was detected in case the two signals (time series) originating from nearby initial conditions overlapped. The limit cycles detected in the present set of ODEs are special in the sense that while amplitudes and frequencies are the same, a phase difference manifests itself. For strange chaotic attractors, information about initial conditions is lost and two time histories seem to belong to a different model system. These limit cycles carry information about the initial conditions. Therefore, one may use them to extract information about initial conditions. It should be noted that the parameter values of synaptic strengths for PCO (-) are $\alpha_{\text {inh }}=1.1, \alpha_{\text {exc }}=0.8$, and for sustained spiking activity are $\alpha_{\text {inh }}=1.2, \alpha_{\text {exc }}=0.8$, whereas for PCO $(+)$ are $\alpha_{\text {inh }}=$ $1.3, \alpha_{\text {exc }}=0.8$. The persistent increase in synaptic strength is caused by a subtype of glutamate receptor N-methyl-Daspartate (NMDA) receptor. The increase in the synaptic strength results from the long-term potentiating of synaptic transmission in the hippocampus. A critical role of NMDA receptor channels in normal WM function of the prefrontal cortex has been suggested by Wang [17].

The view that stimulus-evoked response potential (ERP) represents the sum of individual events that occur with a 


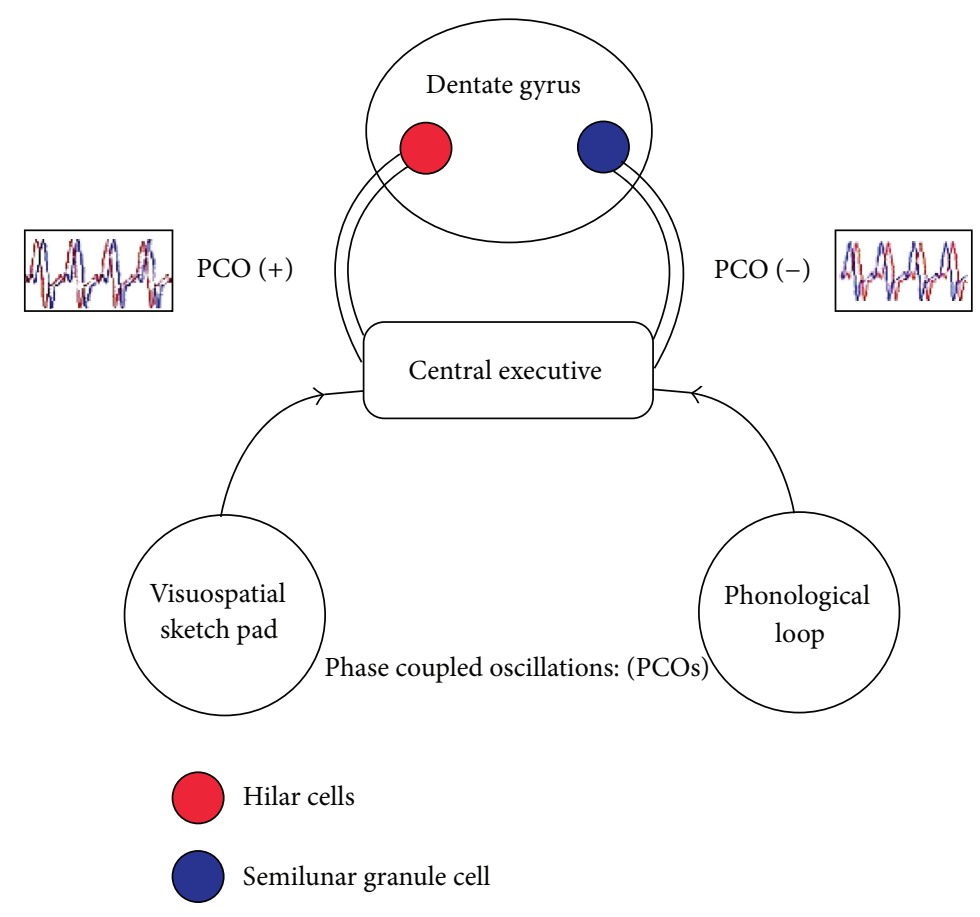

FIGURE 8: Signalling in hippocampus-dependant working memory showing phase-coupled oscillations (PCOs, +, -). Parameter values of the synaptic strengths for PCOs (+) are $\alpha_{\text {inh }}=1.3, \alpha_{\text {exc }}=0.8$. For PCOs (-) they are $\alpha_{\text {inh }}=1.1, \alpha_{\text {exc }}=0.8$.

fixed latency and polarity was reinforced from experiments by Makeig et al. [18], Ermentrout [19], and Jeong and Gutkin [20]. In this context, phase-coupled oscillations assume special significance. The phase-coupled oscillations presumably have their origins in stimulus-triggered response of different neurotransmitters. These oscillations travel together from point of genesis to the point where the response is processed. An alternative way of understanding these phase-coupled oscillators is based on single-neurotransmitter excited neuronal oscillations as a spurt of initial response to perception. Thus phase-coupled limit cycles in hippocampus region can have an impact on present understanding of information processing with relation to time and event processing.

The functioning of the central executive is best understood in terms of different interactions between networks of regions. The neuroimaging studies of the cerebral substrates of the central executive component of the WM model demonstrate that different executive functions recruit various frontal areas and also depend on parietal regions [21-23]. Thus, the phase-preserved oscillations are a time-resolved, coupled phenomenon to process the information/perception dependent on the electrical strength of the signal. The neuronal oscillations travel to the brain through neuronal networks, and the phase-ingrained signal which is dual in nature gets resolved later as classified information. The phase is retained at all times and gives an impression of time surfacing one after the other in the information processing. We propose that these new kinds of phase-coupled oscillations encode information and carry it to the central executive for processing. Figure 8 shows the role of two kinds of PCOs in cell-to-cell signalling in hippocampusdependent working memory. The brain itself is working as a mother board on an autofeedback mechanism sustained internally by the leverage from factors playing part which could be the internal ones in the chemobiological domain. The autofeedback is dependent on the processing strength of the brain as a unit filling the lacunae in the working of the unit to generate oscillations. Recently, the experiments conducted by Larimer and Strowbridge [24] have established that Hilar cell assemblies are linked by persistent firing in semilunar granule cells. This firing is triggered by synaptic barrages. Experiments by these authors on rodent brain tissue have opened up a way to create short-term memory in a population of cells in dentate gyrus. They could identify the brain circuit holding the memory. An in-depth analysis of the experimental data compiled by these investigators indicates that populations of Hilar cells encode the stimulus identity. The perforant firing stimulation evokes plateau potentials and persistent firing in Semilunar Granule Cells (SCGs) [25]. The stimulus-evoked up-states in the dentate gyrus represent a potential cellular basis for hippocampus-dependent memory. It has been proposed recently that SCGs in the dentate molecular layer regulate dentate network activity patterns and working memory formation [26]. Our finding of phasecoupled oscillations may find applications in understanding working memory formation in central nervous system which has a large-scale oscillatory distributed network with directional influences. 


\section{Acknowledgments}

The authors thank Drs. Daya Krishan Lobiyal and Pravitha Ramanand for discussions and Professor Raima Larter for making available a few chapters of B. Speelman's doctoral thesis.

\section{References}

[1] S. Daan and C. Berde, "Two coupled oscillators: simulations of the circadian pacemaker in mammalian activity rhythms," Journal of Theoretical Biology, vol. 70, no. 3, pp. 297-313, 1978.

[2] B. Van der Pol and J. Van der Mark, "The heartbeat considered as a relaxation oscillation, and an electrical model of the heart," Philosophical Magazine, vol. 6, pp. 763-775, 1928.

[3] A. M. Dos Santos, S. R. Lopes, and R. L. Viana, "Rhythm synchronization and chaotic modulation of coupled Van der Pol oscillators in a model for the heartbeat," Physica A, vol. 338, no. 3-4, pp. 335-355, 2004.

[4] J. Hasty, D. McMillen, F. Isaacs, and J. J. Collins, "Computational studies of gene regulatory networks: in numero molecular biology," Nature Reviews Genetics, vol. 2, no. 4, pp. 268-279, 2001.

[5] G. Buzsáki and A. Draguhn, "Neuronal olscillations in cortical networks," Science, vol. 304, no. 5679, pp. 1926-1929, 2004.

[6] Z. Sands, A. Grottesi, and M. S. Sansom, "Voltage-gated ion channels," Current Biology, vol. 15, no. 2, pp. R44-R47, 2005.

[7] W. J. Freeman, "Tutorial on neurobiology: from single neurons to brain chaos," International Journal of Bifurcation and Chaos, vol. 2, pp. 451-482, 1992.

[8] A. L. Hodgkin and A. F. Huxley, "The components of membrane conductance in the giant axon of Loligo," The Journal of Physiology, vol. 116, no. 4, pp. 473-496, 1952.

[9] C. Morris and H. Lecar, "Voltage oscillations in the barnacle giant muscle fiber," Biophysical Journal, vol. 35, no. 1, pp. 193213, 1981.

[10] S. R. Nadar and V. Rai, "Transient periodicity in a morris lecar neural system," ISRN Biomathematics, vol. 2012, Article ID 546315, 7 pages, 2012.

[11] G. A. Carpenter, "A geometric approach to singular perturbation problems with applications to nerve impulse equations," Journal of Differential Equations, vol. 23, no. 3, pp. 335-367, 1977.

[12] N. Fenichel, "Geometric singular perturbation theory for ordinary differential equations," Journal of Differential Equations, vol. 31, no. 1, pp. 53-98, 1979.

[13] E. M. Izhikevich, "Neural excitability, spiking and bursting," International Journal of Bifurcation and Chaos in Applied Sciences and Engineering, vol. 10, no. 6, pp. 1171-1266, 2000.

[14] E. M. Izhikevich, Dynamical Systems in Neuroscience: the Geometry of Excitability and Bursting, MIT Press, Cambridge, Mass, USA, 2007.

[15] M. Colombo and C. G. Gross, "Responses of inferior temporal cortex and hippocampal neurons during delayed matching to sample in monkeys (Macaca fascicularis)," Behavioral Neuroscience, vol. 108, no. 3, pp. 443-455, 1994.

[16] N. Axmacher, C. E. Elger, and J. Fell, "Working memory-related hippocampal deactivation interferes with long-term memory formation," Journal of Neuroscience, vol. 29, no. 4, pp. 1052-1060, 2009.
[17] X. J. Wang, "Synaptic basis of cortical persistent activity: the importance of NMDA receptors to working memory," Journal of Neuroscience, vol. 19, no. 21, pp. 9587-9603, 1999.

[18] S. Makeig, M. Westerfield, T. P. Jung et al., "Dynamic brain sources of visual evoked responses," Science, vol. 295, no. 5555, pp. 690-694, 2002.

[19] B. Ermentrout, "Type I membranes, phase resetting curves, and synchrony," Neural Computation, vol. 8, no. 5, pp. 979-1001, 1996.

[20] H. Y. Jeong and B. Gutkin, "Synchrony of neuronal oscillations controlled by GABAergic reversal potentials," Neural Computation, vol. 19, no. 3, pp. 706-729, 2007.

[21] F. Collette and M. Van Der Linden, "Brain imaging of the central executive component of working memory," Neuroscience and Biobehavioral Reviews, vol. 26, no. 2, pp. 105-125, 2002.

[22] A. D. Baddeley, "Short-term memory for word sequences as a function of acoustic, semantic and formal similarity," The Quarterly Journal of Experimental Psychology, vol. 18, no. 4, pp. 362-365, 1966

[23] A. D. Baddeley, Working Memory, Oxford University Press, Oxford, UK, 1986.

[24] P. Larimer and B. W. Strowbridge, "Representing information in cell assemblies: persistent activity mediated by semilunar granule cells," Nature Neuroscience, vol. 13, no. 2, pp. 213-222, 2010.

[25] D. D. Fraser and B. A. MacVicar, "Cholinergic-dependent plateau potential in hippocampal CA1 pyramidal neurons," Journal of Neuroscience, vol. 16, no. 13, pp. 4113-4128, 1996.

[26] A. Gupta, F. S. Elqammal, A. Proddutur, S. Shah, and V. Shantakumar, "Decrease in tonic inhibition contributes to increase in dentate semilunar granule cell excitability after brain injury," Journal of Neuroscience, vol. 32, no. 7, pp. 2523-2537, 2012. 


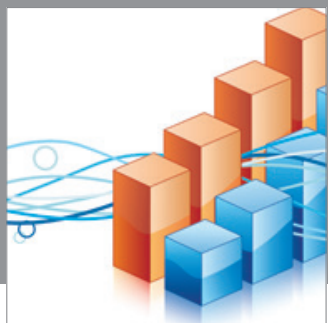

Advances in

Operations Research

mansans

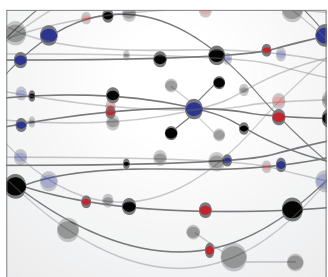

The Scientific World Journal
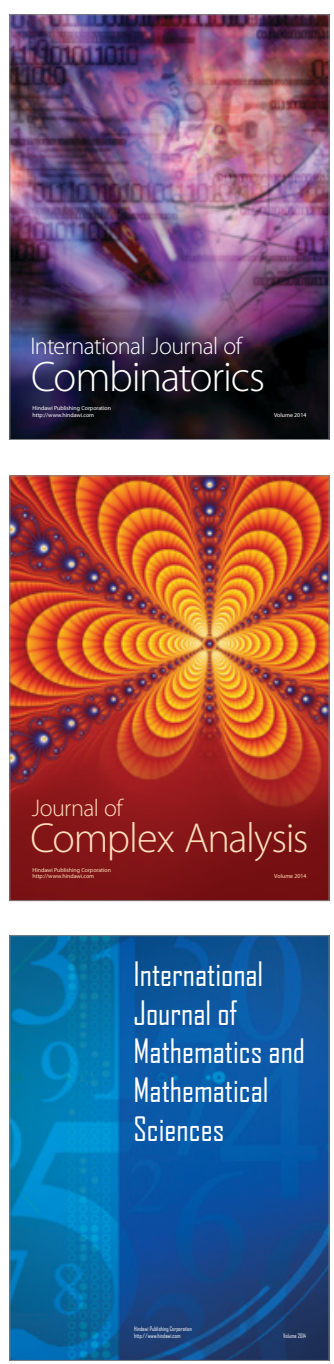
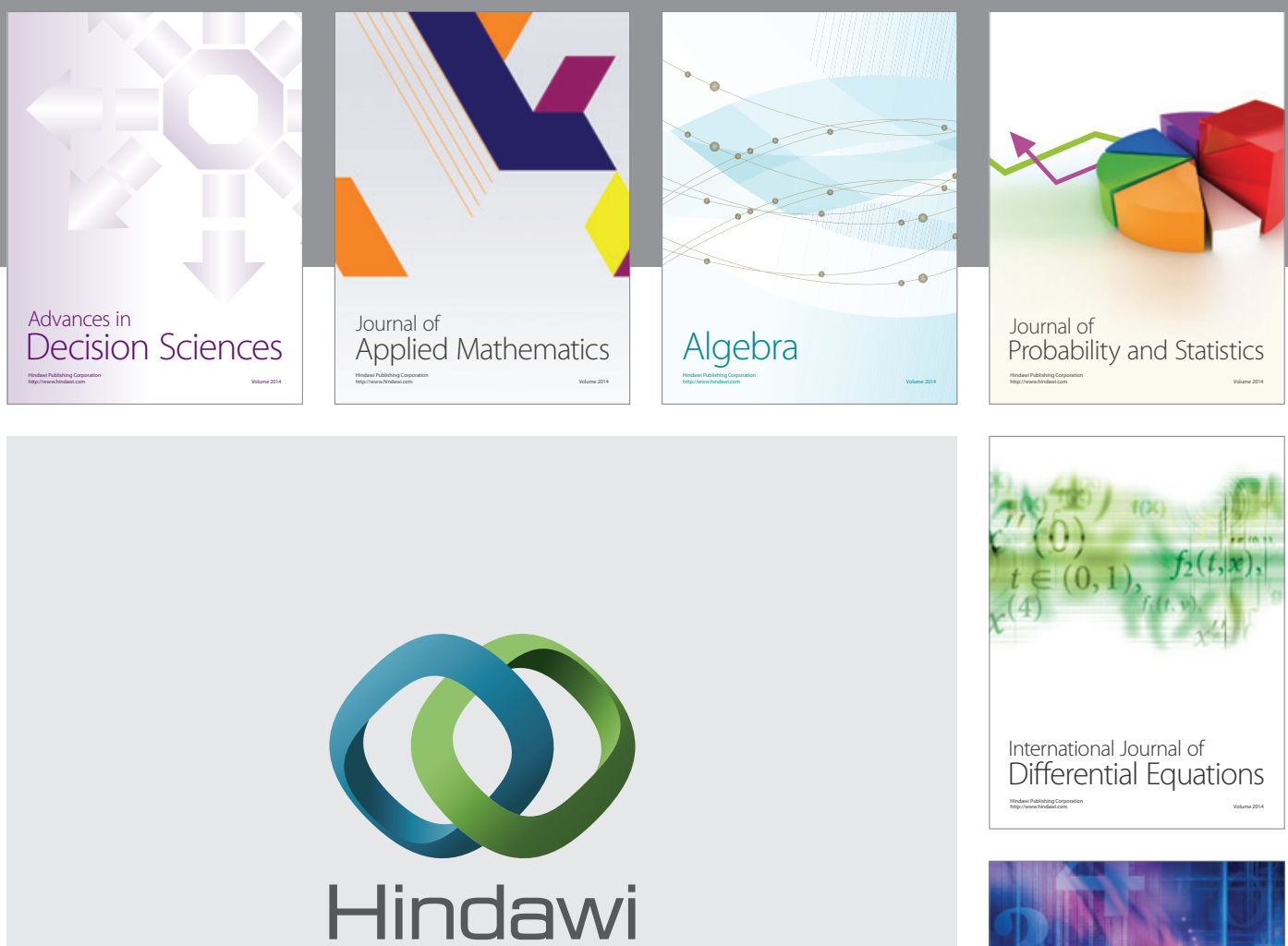

Submit your manuscripts at http://www.hindawi.com
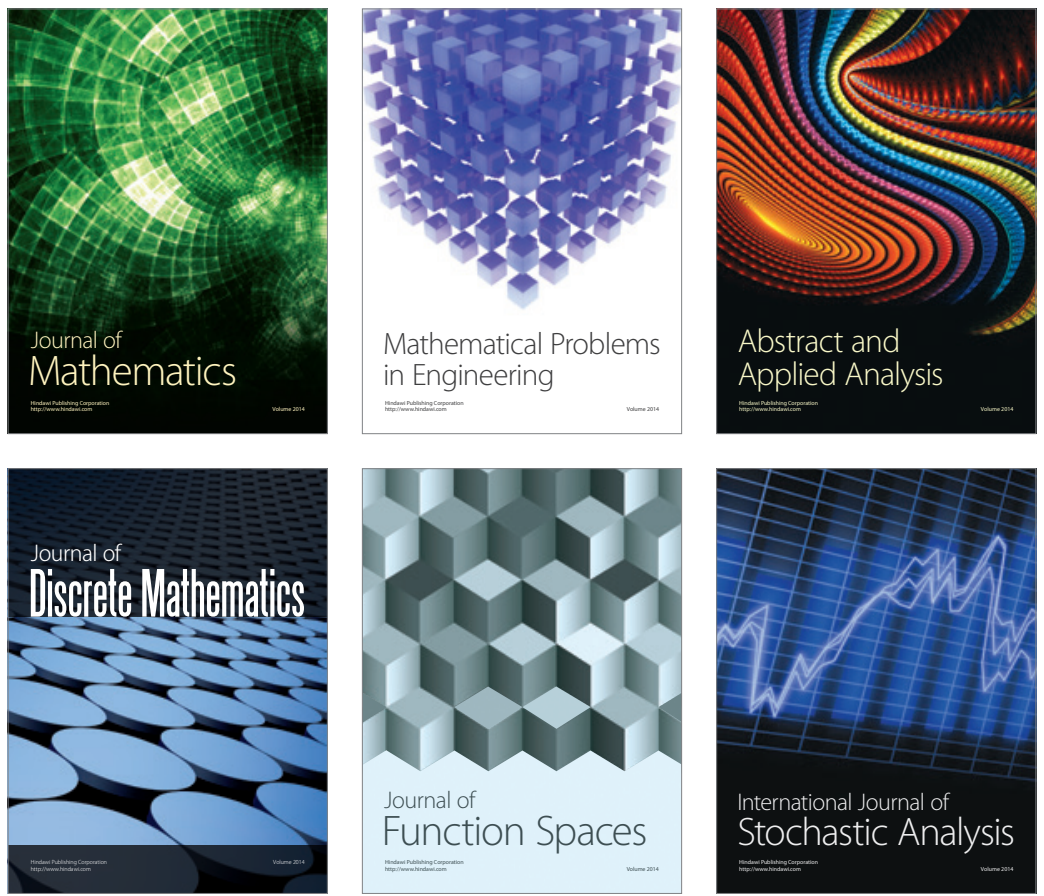

Journal of

Function Spaces

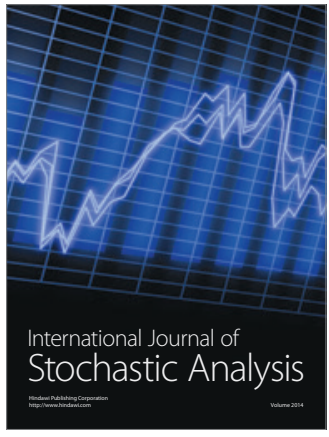

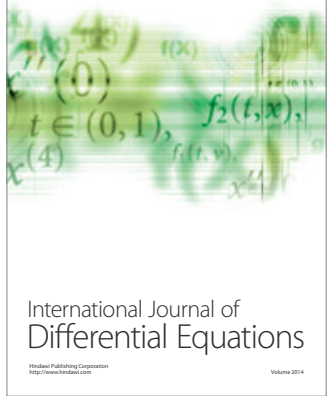
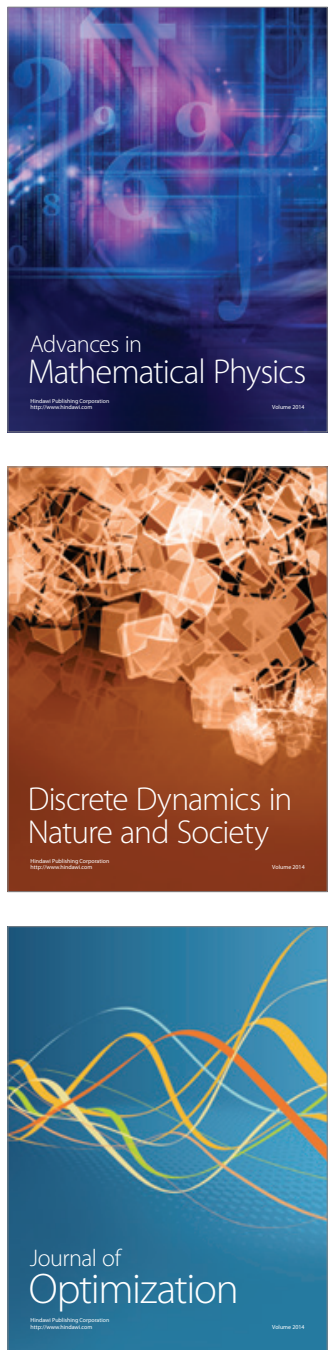